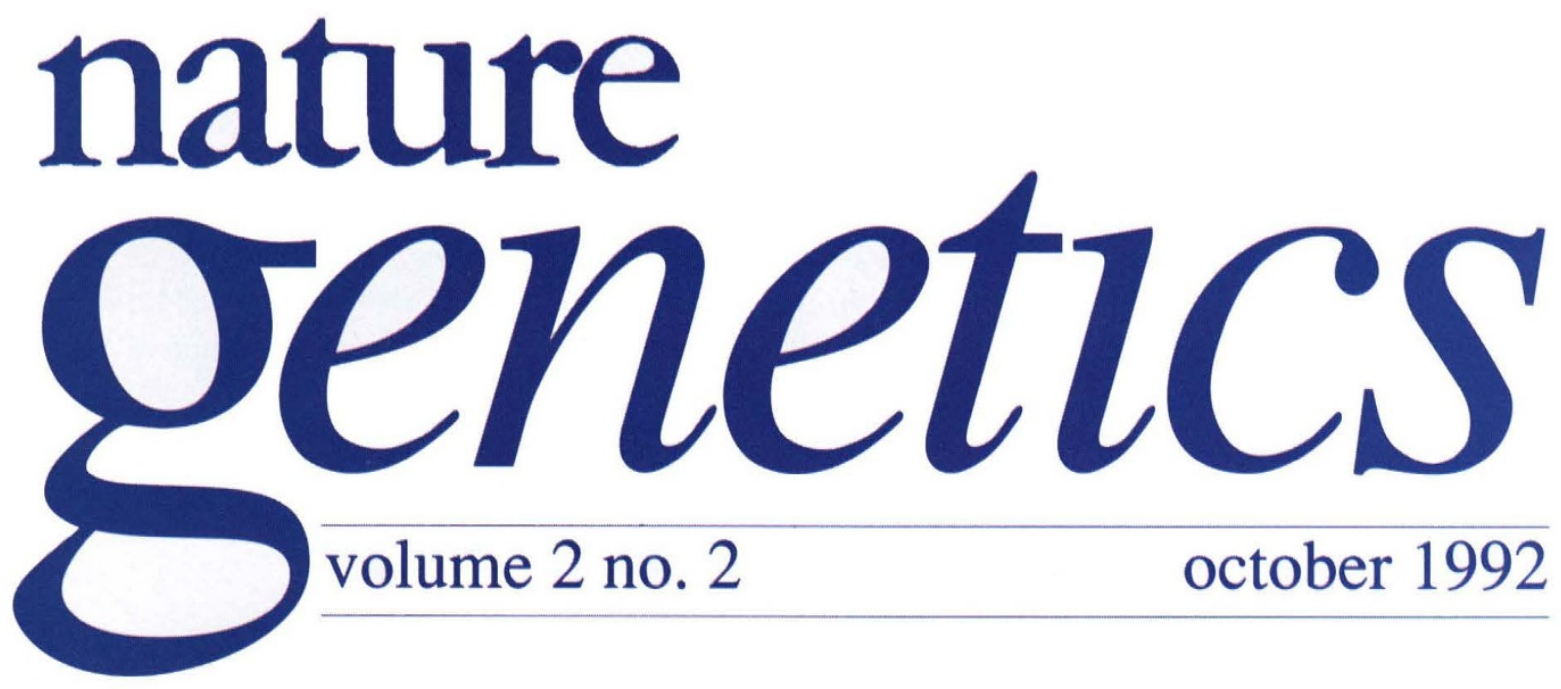

\title{
Keeping track of the translocations
}

The voluminous literature describing the 100 or more chromosomal translocations associated with carcinogenesis ${ }^{1,2}$ is undergoing a modest but notable expansion. A series of recent reports.5, including one in this issue $e^{4}$ on page 113 , adds several interesting new members to the list of genes known to be disrupted or fused in various cancers including leukaemias and solid tumours.

The translocations in leukaemias and lymphomas have traditionally been much better characterized at the molecular level than have solid tumours. The leukaemia translocations frequently disrupt genes that encode important developmental gene regulators, such as helix-loophelix and homeobox proteins, and transcription factors. In many instances, chimaeric fusion proteins may result whose ectopic expression in inappropriate cell-types can lead to the onset of carcinogenesis ${ }^{1,2}$. A handful of such fusion proteins have been characterized: for example, the translocation between the long arms of human chromosomes 15 and 17 - designated $\mathrm{t}(15 ; 17)(\mathrm{q} 21 ; \mathrm{q} 11-12)$ - in a form of acute myeloid leukaemia (AML), results in the fusion of the $\mathrm{N}$ terminus of PML, a zinc-finger protein, to the $\mathrm{C}$ terminus of the retinoic acid receptor $\alpha$ gene. This chimaeric transcription factor is likely to modify the regulation of genes including homeobox genes involved in myeloid differentiation. Another well-characterized example in acute pre-B cell lymphoblastic leukaemia (ALL) is $\mathrm{t}(1 ; 19)(\mathrm{q} 23 ; \mathrm{p} 13)$, which involves the homeoboxgene $P B X$ on chromosome 1 and from chromosome 19 the E2A gene - a basic helix-loop-helix gene that encodes two immunoglobulin enhancer binding proteins. The resulting chimaeric gene product contains the homeobox domain of $P B X 1$ in place of the DNA binding region of $E 2 A$, and again leads to aberrant expression of a DNA binding domain.

Recent work from Thomas Look and colleagues at St Jude Children's Research Hospital in Memphis, Tennessee, has identified a new partner for $E 2 A$ in the chromosome 19p13 translocations in B-cell $\mathrm{ALL}^{3}$. Look and colleagues examined DNA from two patients with a $t(17 ; 19)$ (q22;p13) translocation and cloned the disrupted gene on chromosome 17. They isolated a gene, designated $H L F$ (hepatic leukaemia factor), that encodes a 295 amino acid polypeptide expressed predominantly in the liver and kidney, but not normally in lymphoid cells. The fusions transpose the C-terminal 71 amino acids of HLF onto the bulk of the E2A protein in frame, with the only difference between the two patients being the presence of a short joining fragment in one of them. HLF bears significant homology to the basic region leucine zipper family of transcription factors, and in particular a recently defined class of genes that contains an additional conserved region of some 60 residues upstream of the basic domain. This group includes the thyrotroph embryonic factor ${ }^{6}$ (thought to be required for thyroid stimulating hormone expression in the developing anterior pituitary gland) and the albumin D-box binding protein (necessary for albumin expression in hepatocytes), and now $H L F$. Further studies should demonstrate whether 
the E2A-HLF chimaeric protein promotes B-cell neoplasia by causing the inappropriate expression of HLF-regulated genes.

A commonly observed chromosomal rearrangement in acute leukaemias, especially in young children, occurs at chromosome 11q23. Reciprocal translocations with several other chromosomes have been described for both ALL (including $t(4 ; 11)$ ) andAML (such as $t(9 ; 11)$ ). Groups led by Carlo Croce at the Thomas Jefferson University in Philadelphia and Janet Rowley at the University of Chicago have detected large 1112 kilobase $(\mathrm{kb})$ transcripts derived from a gene spanning the $11 \mathrm{q} 23$ breakpoint, which are upregulated in 11q23 translocations?.

On page 113 of this issue ${ }^{4}$ Glen Evans of the Salk Institute - a prominent figure in the effort to develop a complete physical map of human chromosome 11 - and his colleagues present their studies of the 11q23 region which include a partial characterization of this disrupted gene. The Salk group isolated single-copy sequences derived from cosmids that flank the breakpoint, which in turn detected evolutionarily conserved sequences in various species, and an $11.5 \mathrm{~kb}$ human messenger RNA in lymphocytes and other tissues. (A smaller $4.4 \mathrm{~kb}$ transcript was also detected with some of these unique sequence probes, reminiscent of the complex pattern of expressed transcripts noted by others ${ }^{7}$.)

The genomic sequences immediately flanking the $11 \mathrm{q} 23$ breakpoint revealed a dense clustering of $A l u$ repetitive elements. Nestling just beyond the repeats, however, a few kilobases on either side of the translocation, were two open reading frames that constitute part of the $11.5 \mathrm{~kb}$ transcript and reveal significant homology to the trithorax $(\operatorname{trx})$ gene of Drosophila. Trithorax is a putative transcription factor containing several zinc finger DNA binding domains ${ }^{8}$. Trx is known to bind to homeotic genes in the bithorax complexincluding Ultrabithorax, and is absolutely required throughout early development for differentiation of the head, thorax and abdomen. It is too early to ascertain if the human trx homologue is deregulated or possibly contributes to a chimaeric fusion protein - conflicting evidence has been presented regarding the transcript size variations in different leukaemia patients - but it is interesting that similar genes have previously been implicated in leukaemia. In B-cell ALL, PBX1 becomes part of fusion gene, whereas the HOX11 homeobox gene on chromosome 10 is deregulated in patients with $t(10 ; 14) \mathrm{T}$-cell ALL ${ }^{1,2}$.

Examples such as the trithorax-like translocation should also provide a basis for interesting comparative studies between humans and Drosophila. Another Drosophila-related gene, implicated in $\mathrm{t}(7 ; 9)(\mathrm{q} 34 ; \mathrm{q} 34) \mathrm{T}$-cell ALL, is TAN$1^{9}$, which is a member of the Notch gene family (whose human counterparts are incidentally the subject of the report on page 119 of this issue $\left.{ }^{\mathrm{i} 0}\right)$. $T A N-1$ is highly expressed in lymphoid tissues and is prematurely truncated in three patients with T-cell ALL ${ }^{9}$. Other examples seem destined to emerge soon.

In addition to the continued progress in characterizing haematological tumours, the molecular basis underlying some solid tumours is also emerging. Ewing's sarcoma results from a $t(11 ; 22)(q 24 ; q 12)$ translocation and the ensuing fusion of two genes ${ }^{5}$. The EWS gene on chromosome 22 encodes a domain that resembles the RNA-binding moiety of several proteins. In Ewing's sarcoma, this domain is replaced by the DNA binding ETS-domain of the human homologue of the mouse Fli-1 (Hum-FLI-1) gene, which is itself implicated in erythroleukaemia ${ }^{11}$, suggesting that abnormal regulation of HumFLI-1-controlled genes may be responsible for tumorigenesis. The production of a chimaeric oncogene in papillary thyroid carcinoma caused by a paracentric inversion of chromosome $10 \mathrm{q}$ was described recently ${ }^{12}$, as was the rearrangement of the transcription factor gene $C H O P$ in $t(12 ; 16)(q 13 ; p 11)$ myxoid liposarcomas ${ }^{13}$. As more examples come to light, further insights into the many stages of signal transduction disrupted by such gene rearrangements will emerge, with prospects for enhanced understanding of development, especially in specific cell lineages, and perhaps new therapeutic strategies.

1. Rabbits, T.H. Cell 67, 641-644 (1991).

2. Solomon, E., Borrow, J. \& Goddard, A.D. Science 254, 1153-1160 (1991).

3. inaba, T. et al. Science 257, 531-534 (1992).

4. Djabali, M. et al. Nature Genet. 2, 113-118 (1992).

5. Delattre, O. et al. Nature 359, 162-165 (1992).

6. Drolet, D.W. et al. Genes Devi. 5, 1739-1753 (1991).

7. Ziemin-van der Poel, S. et al. Proc. natn. Acad. Sci. U.S.A. 88, 10735-10739 (1991).

8. Mazo, A.M. et al. Proc. natn. Acad. Sci. U.S.A. 87, 21122116 (1990).

9. Ellisen, L.W. et al. Cell 66, 649-661 (1991).

10. Stifani, S. et al. Nature Genet. 2, 119-127 (1992).

11. Ben-David, Y. et al. Genes Devl. 5, 908-918 (1991).

12. Pierotti, M.A. et al. Proc. natn. Acad. Sci. U.S.A. 89, 16161620 (1992).

13. Aman, P. et al. Genes Chr. Cancer 5, 1-8 (1992). 